\title{
Desarrollo de la Fraseología Especializada en Brasil
}

\author{
Cleci Regina Bevilacqua ${ }^{1}$ \\ ${ }^{1}$ Universidade Federal do Rio Grande do Sul, Porto Alegre, Brasil \\ cleci.bevilacquaeufrgs.br
}

\begin{abstract}
This article presents an overview of research related to Specialized Phraseology conducted in Brazil in recent years. Various denominations and definitions with respect to our object of study, namely Specialized Phraseological Units (SPU), were collected from Brazilian academic publications (dissertations, theses, specialized journals, books, events, etc.) and systematized. As a synthesis, the two identified types of SPU and their denominations were organized into a concept map. Applications (terminographical products, semi-automatic identification) and the thematic areas studied (Medicine, Environmental Law, Economics, etc.) are also presented. The justification for the study is grounded in the need to find and gather information about the research carried out within the scope of Specialized Phraseology, which allowed us to draw an initial overview of the studies in Brazil. As a result, we hope to be able to disseminate Brazilian scientific publications on the subject.
\end{abstract}

Keywords: Specialized Phraseology, Specialized Phraseological Units, Brazilian Research.

\section{Introducción}

El presente trabajo presenta un panorama de las investigaciones relativas a la Fraseología Especializada llevadas a cabo en Brasil. Dichas investigaciones empiezan a desarrollarse a finales de los años de 1990, motivadas sobre todo por la introducción de la Terminología, ámbito en el que se suele incluir la Fraseología Especializada. Eso ocurrió principalmente por la consolidación de grupos de investigación y por la inserción de disciplinas de Terminología en cursos de pregrado y posgrado en Traducción.

Considerando estos aspectos, a partir de la compilación y revisión de la producción científica brasileira de los últimos 15 años, se pudo identificar la existencia de diferentes perspectivas (Terminología, Traducción, Lingüística de Corpus y Procesamiento del Lenguaje Natural) en los estudios relativos al tema y a su objeto de estudio - las Unidades Fraseológicas Especializadas (UFEs) -, que generan distintas denominaciones y definiciones para dicho fenómeno. Se busca identificar esas denominaciones y definiciones asociándolas a sus autores y a los temas y áreas tratados.

Inicialmente, se hace referencia a las fuentes de recogida de las informaciones. Enseguida, se sistematizan las distintas denominaciones y definiciones relativas a las UFEs, buscando mostrar los diferentes tipos y sus principales características. Como síntesis de esta etapa, se presenta un mapa conceptual con la síntesis de los resultados 
obtenidos. También se muestra la aplicación de los estudios realizados en el área y a los temas estudiados.

Algunas de las justificaciones que sostienen el trabajo son: el interés por ese tema para la traducción y producción de textos especializados, así como la extracción de UFEs a partir de corpus especializados y su aplicación en la enseñanza de la traducción y de lenguas para propósitos específicos; la ausencia de un relevamiento sobre las investigaciones realizadas en Brasil. Se espera poder contribuir a la construcción de dicho panorama, aunque de forma inicial, y divulgar la producción científica brasileña relativa a la Fraseología Especializada.

\section{Las fuentes para la recogida de los datos}

Para la recogida de los datos, se hizo un relevamiento general en el Portal de Periódicos de Capes, en repositorios digitales de universidades y en Google, utilizándose palabras clave como colocação(ões) especializada(s), combinatórias léxicas especializadas, expressões multipalavras, expressões recorrentes, fraseologia especializada, fraseologismos, unidades fraseológicas especializadas. También se buscaron informaciones en páginas web de proyectos y de grupos de investigación, asociaciones, eventos del área y de áreas afines, libros y revistas nacionales relacionados al estudio del léxico. Consideramos, además, nuestro conocimiento del área y el contacto con los investigadores brasileños.

Algunas de las fuentes consultadas fueron: Universidade de Brasília (UnB), Universidade Estadual Paulista Júlio Mesquita, campus de São José do Rio Preto (UNESP), Universidade Estadual do Ceará (UECE), Universidade Federal do Mato Grosso do Sul (UFMS), Universidade Federal do Rio Grande do Sul (UFRGS), Universidade de São Paulo (USP); proyecto COMET (USP) $)^{1}$, Projeto Terminológico Cone Sul (TERMISUL, UFRGS) ${ }^{2}$, TexQuim/TexTECC(UFRGS) ${ }^{3}$, Grupo de Trabalho em Lexicologia, Lexicografia e Terminologia da Associação Nacional de Pesquisa em Letras e Linguística (GTLex-ANPOLL) ${ }^{4}$, Rede Ibero-americana de Terminologia (RITerm) e Rede Panlatina de Terminologia (REALITER) ${ }^{5}$; Congresso Internacional de Fraseologia e Paremiologia, Encontro Nacional de Tradutores, Encontro de Linguística de Corpus (ELC); libros (Avanços na Linguística de Corpus no Brasil; TAGNIN; VALE, 2008; Tendências atuais na pesquisa descritiva e aplicada em fraseologia e paremiologia; ALVAREZ, 2012); publicaciones periódicas (Alfa ${ }^{6}$, Debate Terminológico ${ }^{7}$, DomíniosdaLingu@gem ${ }^{8}$,TradTerm ${ }^{9}$ ).

\footnotetext{
${ }^{1}$ http://comet.fflch.usp.br/

${ }^{2} \mathrm{http}: / /$ www.ufrgs.br/termisul/

${ }^{3}$ http://www.ufrgs.br/textecc/textquim/; http://www.ufrgs.br/textecc/

${ }^{4}$ http://www.letras.ufmg.br/gtlex/

${ }^{5}$ https://sites.google.com/view/riterm/riterm; http://www.realiter.net/

${ }^{6} \mathrm{http}: / /$ seer.fclar.unesp.br/alfa

${ }^{7} \mathrm{http}: / /$ seer.ufrgs.br/riterm

${ }^{8}$ http://www.seer.ufu.br/index.php/dominiosdelinguagem

${ }^{9} \mathrm{http}: / / \mathrm{www}$. revistas.usp.br/tradterm
} 


\section{Las UFEs: denominaciones y definiciones}

El análisis de los datos permitió identificar distintas denominaciones y definiciones para las UFEs, que se agruparon en dos grandes grupos descritos a continuación.

1) UFEs caracterizadas por estructuras semejantes a las colocaciones, es decir, están formadas por una base y un colocado (la unidad que acompaña la base) y pueden describirse según determinadas estructuras morfosintácticas. Esta perspectiva se basa sobre todo en la propuesta de colocación de Hausmann $(1979,1990)$ para las colocaciones de la lengua general y de L'Homme $(1998,2000)$ y de L'Homme y Bertrand (2000) para los lenguajes de especialidad. Algunos ejemplos son: adquirir ações, ações subscritas (ORENHA-OITTANO, 2009), absorver calor, absorção de calor, calor absorvido (BEVILACQUA, 2004). La revisión de los autores mostró que hay varias denominaciones utilizadas para este tipo de UFE: colocação especializada, UFEs, UFEs eventivas, Combinatórias Léxicas Especializadas (CLES) y expressões recorrentes.

Algunos de los investigadores siguen esta perspectiva son: Bertonha e Zavaglia (2015), Bevilacqua (1999, 2004); Bevilacqua et al (2013), Castanho (2011),Cruz (2011), Esperandio (2015), Ferreira (2015), Leipnitz (2013), Orenha-Ottaiano (2009), Pacheco (2015), Santiago (2013),Waquil (2013) y Zilio (2009).

2) UFEs caracterizadas como fórmulas, que pueden llegar a frases autónomas o a párrafos completos. Esta perspectiva se fundamenta en las propuestas de autores como Roberts (1994-1995), Parc (1993) y Gouadec (1994). Algunos ejemplos pueden ser: $O$ não cumprimento de $[\mathrm{x}]$ sujeita $[\mathrm{y}]$ a [z], donde [x] puede ser lei, decreto, parágrafo; [y] a los infrator(es) y [z] a las punições (medida, cautelar, advertência, multa, penalidades). Las denominaciones encontradas fueron: colocações especializadas estendidas, combinatórias léxicas especializadas jurídicas, matrizes fraseológicas sem pivô terminológico. Algunos de los autores que siguen esta perspectiva son: Bevilacqua (1996), Tagnin (2012), Orenha-Ottaiano (2009).

La síntesis de los tipos y denominaciones de UFEs se encuentra en el mapa conceptual presentado en la Figura 1.

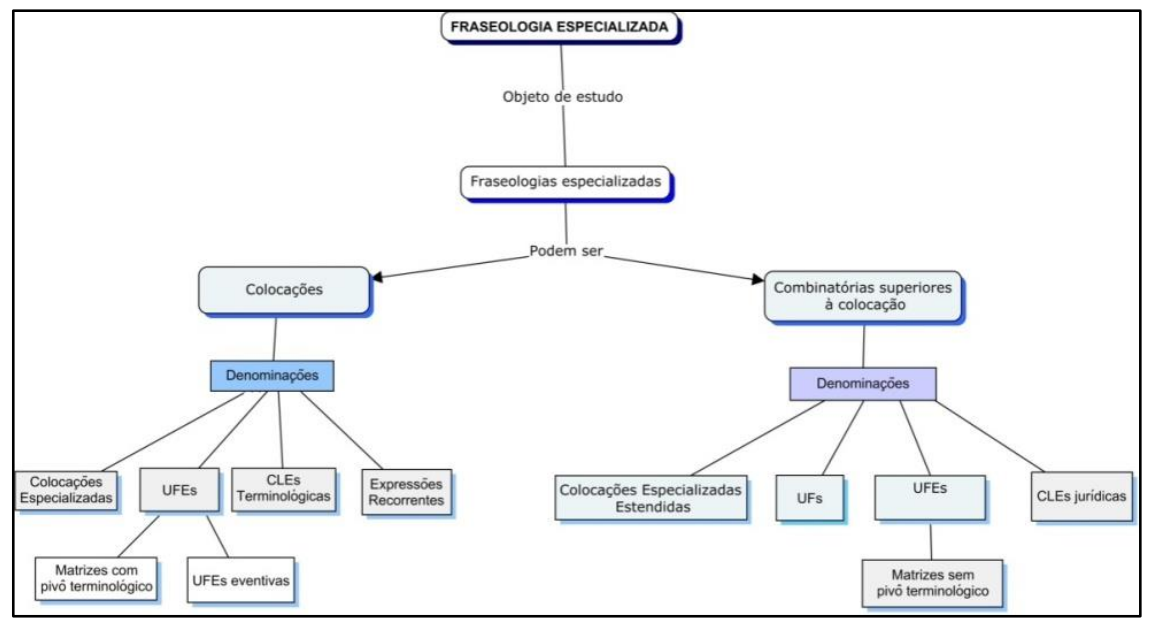

Fig. 1 - Mapa conceptual: tipos y denominaciones de UFEs. 


\section{Las aplicaciones, temas y áreas investigados}

Respecto de las aplicaciones se identificaron dos tendencias: una dirigida a la aplicación y otra a la descripción. Para la primera las temáticas evidencian la interfaz entre: a) la Fraseología Especializada y la Lingüística de Corpus, que trata temas como la constitución de corpus y las herramientas para la extracción e identificación de las UFEs y la búsqueda de equivalentes de las UFEs en lenguas extranjeras; b) la Fraseología Especializada y la Traducción, en la que se destacan temas como la relevancia de las UFEs en el proceso de traducción, las propuestas de diseño de recursos terminológicos para traductores (glosarios, bases de datos) y la identificación de equivalentes en las lenguas de trabajo del traductor.

En lo que se refiere a la descripción, los temas tratados son: la aplicación de modelos lingüísticos para la descripción de las UFEs, aspectos diacrónicos; descripción sintáctico-semántica; equivalencia del portugués para distintas lenguas extranjeras; metáforas, patrones léxico-gramaticales.

Las áreas temáticas tratadas en los trabajos analizados se refieren a: Cardiología, contratos y reglamentos sociales, Culinaria, Derecho Ambiental, Derecho Comercial internacional, Economía, Educación, Educación a Distancia, exportación, fútbol, Medicina, Pediatría, publicidad, Traducción, Traducción Pública, Turismo, etc.

Los datos permitieron destacar que hay una interrelación entre la Fraseología Especializada con los Estudios de Traducción y la Lingüística de Corpus, estableciendo un interdisciplinariedad entre dichas áreas. Asimismo, se puede afirmar que la Fraseología Especializada también es transdisciplinar puesto que se aplica a varios ámbitos del saber - Derecho, Medicina, Economía, etc.

\section{Conclusiones}

A partir de las informaciones recogidas y de su sistematización se buscó presentar un panorama inicial de la Fraseología Especializada en Brasil. El análisis de los datos reveló que hay una diversidad denominativa para referirse a las UFEs y que, aunque sus propiedades sean comunes y sus conceptos sean muy cercanos, se identificaron dos grandes categorías establecidas principalmente por sus estructuras: las colocaciones especializadas y las fórmulas. Esas categorías están conformes a las propuestas de autores reconocidos en el área, tanto los que se dedican a la fraseología de la lengua general como a la fraseología especializada. Para cada categoría se indicaron sus denominaciones y los autores brasileños. Además, se identificaron las aplicaciones, temáticas y áreas sobre las que se estudian las UFEs, mostrando que la Fraseología Especializada es a la vez interdisciplinar y transdisciplinar.

Con el conjunto de datos relevados, se puede decir que hay una producción académica significativa sobre la Fraseología Especializada en Brasil y la búsqueda por nuevos datos puede revelar que esa producción quizás sea aún mayor. Sin embargo, parece ser que la sistematización de los datos encontrados permitió construir una visión general inicial de los estudios del área en Brasil y, a la vez, posibilitó organizar la terminología utilizada para referirse a las UFEs. 


\section{Referencias}

1. Alvarez, M. L. O. (ed.): Tendências atuais na pesquisa descritiva e aplicada em fraseologia e paremiologia, vol. I e II. Pontes, São Paulo (2012).

2. Bevilacqua, C. R.: A fraseologia jurídico-ambiental. Tesis. (Maestría en Estudios del Lenguaje). Programa de Posgrado en Letras, Instituto de Letras, UFRGS, Porto Alegre (1996).

3. Bevilacqua, C. R.: Unidades fraseológicas especializadas: estado de la cuestión y perspectivas. Tesina. (Doctorado en Lingüística Aplicada - Léxico). Institut Universitari de Lingüística Aplicada, Universitat Pompeu Fabra, Barcelona (1999).

4. Bevilacqua, C. R.: Unidades Fraseológicas Especializadas Eventivas: descripción y reglas de formación en el ámbito de la energía solar. Tesis. (Doctorado en Lingüística Aplicada - Léxico). Institut Universitari de Lingüística Aplicada, Universitat Pompeu Fabra, Barcelona (2004).

5. Bevilacqua, C. R. et al: Combinatórias Léxicas Especializadas da Linguagem Legislativa: uma abordagem orientada pelo corpus. En: Murakawa, C., Nadin, O. L. (eds.) Terminologia: uma ciência interdisciplinar, pp.227-243. Cultura Acadêmica, São Paulo (2013).

6. Bertonha, F. H. C., Zavaglia, C.: Dicionário bilíngue de fraseologismos jurídicos: uma proposta. En: Zavaglia, C., Stupiello, E. (eds.) Tendências Contemporâneas dos Estudos da Tradução, vol. 2, pp. 36-64. Ed. UNESP, São José do Rio Preto (2015).

7. Castanho, R. M. C.: Proposta para a Elaboração de um Glossário de Colocações na Área Médica - Subárea Hipertensão Arterial. Tesis (Maestría en Estudios Lingüísticos y Literarios en Inglés). USP, São Paulo (2003).

8. Cruz, L. P. R.: Estudo da tradução de colocações especializadas na área de exportação e agronegócios baseado em um corpus paralelo. Tesis (Maestría en Estudios Lingüísticos). UNESP, São José do Rio Preto (2011).

9. Esperandio, I. B.: Colocações na legendagem de seriados: Um estudo exploratório. En: Zavaglia, C. Stupiello, E. (eds.) Tendências Contemporâneas dos Estudos da Tradução, vol. 2, pp. 139-164. UNESP, São José do Rio Preto (2015).

10. Gouadec, D.: Nature et traitement dês entités phraséologiques. Terminologie et phraséologie. En: Acteurs et amenageurs: Actes de la deuxième Universitéd'Automne en Terminologie, pp. 167-193. La Maison du Dictionnaire, Paris (1994).

11. Ferreira, V. R.: Glossário terminológico básico da teoria da tradução: uma experiência com o e-termos. Tesis (Msestría en Estudios del Lenguaje). Universidade Federal do Mato Grosso do Sul, Campo Grande (2015).

12. Hausmann, F. J.: Un dictionnaire de collocationsest-il posible? Travaux de linguistique et de littérature, 17 (1), 187-195 (1979).

13. Hausmann, F. J.: Le dictionnaire de collocations. En: Hausmann, F. J. et al. (eds.) An International encyclopedia of lexicography, vol. 1, p. 1010-1019. Walter de Gruyter, Berlin- New York (1990).

14. Leipnitz, L.: Fraseologias terminológicas no ensino da tradução. En: Tagnin, S. E. O.; Bevilacqua, C. R. (eds.) Terminologia, pp.113-127. HUB, São Paulo (2013). 
15. L'Homme, M-C.: Caractérisation dês combinaisons lexicales spécialisées par raport aux collocations de langue générale. En: Fontenelle, T. et al (eds.) Euralex'98 Proceedings, vol. II, pp. 513-522. Université de Liège, Liège (1998).

16. L'Homme, M-C.: Understanding Specialized Lexical Combinations. Terminology 6(1), 89-110 (2000).

17. L'Homme, M-C., Bertrand, C.: Specialized Lexical Combinations: should they be described as collocations or in terms of selectional restrictions? Página web, http://olst.ling.umontreal.ca/pdf/berlhom.pdf , último acceso 2015/10/15.

18. Orenha-Ottaiano, A.: Unidades fraseológicas especializadas: colocações e colocações estendidas em contratos sociais e estatutos sociais traduzidos no modo juramentado e não-juramentado. Tesis. (Doctorado en Estudios Lingüísticos). UNESP, São José do Rio Preto (2009).

19. Pacheco, S. A.: Configurações sintático-semânticas das unidades fraseológicas especializadas: o caso do léxico do exército brasileiro. Tesis. (Doctorado en Estudios de Lenguaje). Programa de Posgrado en Letras, Instituto de Letras, UFRGS, Porto Alegre (2015).

20. Parc, F.: Traitement de la phraséologie terminologique tirée dês textes législatifs et réglementaires suisses. Terminologies Nouvelles, 10, 115-119 (1993).

21. Roberts, R.: Identifying the phraseology of languages for special purposes (LSPs). Alfa: Actes de langue françaiseet de linguistique, vol. 7/8, pp. 61-69. Universitas Dalhousiana, Halifax, (1994-1995).

22. Santiago, M.S.: Unidades fraseológicas especializadas em tutoriais de ambientes virtuais de aprendizagem: proposta de um sistema classificatório com base na valência verbal. Tesis (Doctorado en Estudios del Lenguaje). Programa de Posgrado en Letras, Instituto de Letras, UFRGS, Porto Alegre (2013).

23. Tagnin, S. E. O.: Fraseologia especializada para tradutores: glossários direcionados pelo corpus. En: Alvarez, M. L. O. (ed.) Tendências atuais na pesquisa descritiva e aplicada em fraseologia e paremiologia, vol. 1, pp. 333-344. Campinas, Pontes (2012).

24. Tagnin, S. E. O., Vale, O. A. (eds.): Avanços da Linguística de Corpus no Brasil. Humanitas, São Paulo (2008).

25. Waquil, M. L.: Tradução de textos especializados: unidades fraseológicas especializadas e técnicas tradutórias. Tesis (Maestría en Estudios del Lenguaje). Programa de Posgrado en Letras, Instituto de Letras, UFRGS, Porto Alegre (2013).

26. Zilio, L.: Colocações especializadas e 'Komposita': um estudo constrastivo alemão-português na área de cardiologia. Tesis. (Maestría en Estudios del Lenguaje). Programa de Posgrado en Letras, Instituto de Letras, UFRGS, Porto Alegre (2009). 